\title{
Investigation of the impact of urine handling procedures on interpretation of urinalysis findings and product safety in subjects treated with ezogabine
}

\author{
This article was published in the following Dove Press journal: \\ Therapeutics and Clinical Risk Management \\ 6 May 2013 \\ Number of times this article has been viewed
}

\author{
Neil Brickel' \\ Sarah DeRossett ${ }^{2}$ \\ Mauro Buraglio 3 \\ Christopher Evans ${ }^{4}$ \\ Siôn Jones ${ }^{5}$ \\ 'Neurosciences Therapy Area \\ Unit, GlaxoSmithKline, Uxbridge, \\ Middlesex, UK; ${ }^{2}$ Neurosciences \\ Therapy Area Unit, GlaxoSmithKline, \\ Research Triangle Park, NC, USA; \\ ${ }^{3}$ Neurosciences Therapy Area \\ Unit, GlaxoSmithKline, Stevenage, \\ Hertfordshire, UK; ${ }^{4}$ Bioanalytical \\ Science and Toxicokinetics, PTS- \\ DMPK, GlaxoSmithKline, King \\ of Prussia, PA, USA; ${ }^{5}$ Global Clinical \\ Safety and Pharmacovigilance, \\ GlaxoSmithKline, Uxbridge, \\ Middlesex, UK
}

\begin{abstract}
Background: Ezogabine (also known by the international nonproprietary name of retigabine) is an antiepileptic drug codeveloped and comarketed by Valeant Pharmaceuticals North America and GlaxoSmithKline, which reduces neuronal excitability by enhancing the activity of potassium channels and has the potential to have effects on the urinary system through a pharmacologic action on bladder smooth muscle. In a single post-herpetic neuralgia trial, but not in an extensive epilepsy development program, proteinuria was unexpectedly reported in patients receiving ezogabine. Consequently, investigations were conducted to determine whether the reported proteinuria represented a true or false-positive finding.
\end{abstract}

Methods: Patients receiving ezogabine 900-1200 mg/day in an open-label extension (Study 303) of a Phase III epilepsy trial voluntarily provided urine samples. Fresh samples were analyzed immediately at the study site, and stabilized aliquots were analyzed 1-3 days after collection at two central laboratories. In an open-label study in healthy volunteers receiving ezogabine 600-900 mg/day (Study RTG114137), urine samples were analyzed fresh ( $<2$ hours after collection) and, using two different stabilizers and storage at room temperature, after 24 and 72 hours. Fluid intake was restricted prior to one sample point. Albumin:creatinine ratios were assessed in both studies.

Results: In Study 303, there was notable variation in clarity, color, and proteinuria between fresh and stored urine samples, and between samples analyzed at different laboratories. In RTG114137, reporting rates of proteinuria were elevated following storage using one stabilizer, and the frequency of color change from fresh to stored samples differed between the stabilizers and between 24 and 72 hours with one stabilizer. Following fluid restriction, proteinuria rates were elevated with both stabilizers. Poor tolerability of ezogabine $750-900 \mathrm{mg} /$ day resulted in limited titration beyond $750 \mathrm{mg}$ /day and early termination of RTG114137.

Conclusion: Hydration status, interval between urine collection and analysis, and the type of stabilizer used are all factors that may lead to false-positive proteinuria findings in patients receiving ezogabine and should be borne in mind if abnormalities are reported.

Keywords: antiepileptic drugs, ezogabine, retigabine, urinary safety, urinalysis

\section{Introduction}

The antiepileptic drug ezogabine (also known by the international nonproprietary name of retigabine), which was codeveloped and is comarketed by Valeant Pharmaceuticals International (Montreal, Quebec, Canada) and GlaxoSmithKline (Research Triangle Park, NC, USA), reduces neuronal excitability by enhancing the activity of KCNQ (Kv7.2-7.5) potassium channels. ${ }^{1}$ Ezogabine is rapidly absorbed and extensively metabolized via

\footnotetext{
Correspondence: Neil Bricke GlaxoSmithKline, Stockley Park West I-3 Iron Bridge Road, Uxbridge, Middlesex UBII IBT, UK

Tel +4402089902582

Fax +44 01865254301

Email neil.r.brickel@gsk.com
}

submit your manuscript | www.dovepress.com

Dovepress

http://dx.doi.org// 0.2147/TCRM.S42536
Therapeutics and Clinical Risk Management 2013:9 207-213

(C) 2013 Brickel et al, publisher and licensee Dove Medical Press Ltd. This is an Open Access article which permits unrestricted noncommercial use, provided the original work is properly cited. 
formation of an $N$-acetyl metabolite and by $N$-glucuronidation of both ezogabine and the $N$-acetyl metabolite. ${ }^{2,3}$ Elimination of ezogabine and its metabolites is predominantly renal, with $30 \%-40 \%$ excreted in urine as the parent drug and a further $40 \%-50 \%$ as metabolites. ${ }^{4}$ Ezogabine $600-1200 \mathrm{mg}$ /day has been shown to be efficacious and generally tolerated as adjunctive therapy in adults with partial-onset seizures in three pivotal controlled trials, ${ }^{5-7}$ and has been approved for adjunctive use in adults with partial-onset seizures by regulatory authorities including the US Food and Drug Administration and the European Medicines Agency.

Ezogabine has a pharmacologic effect on the smooth muscle of the urinary bladder through interaction with KCNQ potassium channels, ${ }^{8-10}$ and consequently has the potential for effects on the urinary system. In the pivotal controlled trials in epilepsy, urinary and renal adverse events were reported more frequently in patients receiving ezogabine than in those receiving placebo (17\% versus $13 \%) .{ }^{11}$ The most common renal and urinary adverse events in the ezogabine group compared with the placebo group were dysuria $(2.3 \%$ versus $0.7 \%)$, urinary hesitation $(2.2 \%$ versus $0.9 \%)$, chromaturia $(1.6 \%$ versus $0.2 \%)$, hematuria (1.6\% versus $0.7 \%)$, and abnormal urine analysis $(1.6 \%$ versus $0.9 \%)$. In addition, urinary tract infection was reported commonly in both groups $(4.7 \%$ versus $4.3 \%$ ). Urinary and renal adverse events, the majority of which were mild, were reported in $26 \%$ of 1365 patients receiving ezogabine in the Phase II-III clinical development program; most of these patients continued treatment. ${ }^{11} \mathrm{O}$ the urinary and renal adverse events reported in the Phase II-III clinical development program, only urinary tract infection was observed in $\geq 5 \%$ of patients receiving ezogabine; in addition, urinary hesitation, abnormal urinalysis, dysuria, urinary retention, hematuria, chromaturia, polyuria, residual urine volume, leukocyturia, and proteinuria were reported in $\geq 1 \%$ of patients receiving ezogabine. ${ }^{11}$ In addition to symptomatic effects on the bladder, changes in some urinalysis parameters were seen in a placebo-controlled Phase IIa safety and efficacy study of ezogabine in patients with post-herpetic neuralgia (NCT00612105; http://www.clinicaltrials.gov).

In the post-herpetic neuralgia study, patients were treated with placebo or ezogabine, and urine specimens were collected during treatment. After collection, a stabilizer was added to the urine, and the specimens were transported to a central laboratory and analyzed within 96 hours of collection using a fully automated urine chemistry analyzer. Incidence rates for proteinuria, haziness/cloudiness, and abnormal coloration were approximately $10 \%, 33 \%$, and $3 \%$, respectively, for patients receiving placebo, and approximately $30 \%$,
$95 \%$, and $50 \%$, respectively, for patients receiving ezogabine (Valeant Pharmaceuticals International, data on file). However, clinically meaningful changes in renal function were not detected and no adverse events were associated with these unexpected urinalysis findings, which resolved completely with gradual withdrawal of ezogabine over 3 weeks (Valeant Pharmaceuticals International, data on file). Proteinuria or urinalysis findings similar to those observed in the post-herpetic neuralgia study were not reported in the pivotal controlled trials, ${ }^{5-7}$ which used a different central laboratory from that in the post-herpetic neuralgia study.

It is well established that urinary crystal formation is common ${ }^{12}$ and cloudy urine may be due to precipitated crystals. ${ }^{13}$ Crystals with a bilirubin-like appearance (but shown not to be bilirubin by chemical analysis) were detected in the urine of $15 \%$ of patients receiving ezogabine in the Phase III studies. ${ }^{11}$ In addition, aqueous solutions of ezogabine are known to develop a bluish-purple color over 24-72 hours. ${ }^{11}$ Hence, it was hypothesized that the urinalysis findings in the post-herpetic neuralgia study resulted from urine handling techniques and/or ex vivo degradation of renally excreted ezogabine and its metabolites, producing urine turbidity and discoloration that interfered with the automated analysis procedures performed by the central laboratory used in the study.

To test the hypothesis that urine handling procedures, storage, and use of preservatives may impact laboratory urinalysis results from subjects receiving repeat doses of ezogabine, two further studies were conducted and the results are presented here.

\section{Materials and methods Study designs Study 303}

Study 303 (NCT00310375) ${ }^{14}$ is an open-label extension of the Phase III RESTORE 1 trial, which evaluated the efficacy and safety of ezogabine 900-1200 mg/day in adults with partial-onset seizures. ${ }^{7}$ A protocol amendment was designed to explore the hypothesis that urinary findings in a placebocontrolled Phase IIa safety and efficacy study of ezogabine in patients with post-herpetic neuralgia (NCT00612105; http:// www.clinicaltrials.gov) were due to sample handling. The amendment enabled patients enrolled in Study 303 to provide urine samples voluntarily for evaluation. Urine specimens were divided into five aliquots: one was analyzed immediately on site and, to enable a direct comparison of different laboratory methodologies on urinalysis outcomes, two central laboratories ( $\mathrm{A}$ and $\mathrm{B}$ ) each received two stabilized aliquots to be tested on arrival (1-3 days after collection). 


\section{Study RTG I I4 I37}

Study RTG114137 was an open-label, repeat-dose study in healthy volunteers aged 40-65 years, specifically designed to explore the underlying mechanisms responsible for the urinalysis observations in the above-mentioned post-herpetic neuralgia study. Subjects were inpatients at the clinical unit for the duration of dosing (Figure 1). Urine (first morning void) collection was scheduled for the fourth day of treatment with ezogabine 600,750, and $900 \mathrm{mg} /$ day (steady state), and for the sixth day of treatment with ezogabine 600 and $900 \mathrm{mg}$ /day following 24-30 hours of fluid restriction. Urine samples were divided into six aliquots. At each time point, one aliquot was analyzed within 2 hours of collection; different stabilizers were added to two aliquots, which were analyzed after 24 hours and 72 hours of storage at room temperature; and one spare aliquot was frozen for further chemical analysis, if considered necessary. The stabilizers used were commonly used brands (stabilizer 1, Stabilur ${ }^{\circledR}$ [Cargille-Sacher Laboratories, Cedar Grove, NJ, USA] and stabilizer 2, BTA urine stabilizer [Bio-Medical Products Corporation, Mendam, NJ, USA]). Storage times were selected to replicate the range of intervals between urine collection and analysis in Study 303.

\section{Assessments}

\section{Study 303}

On-site tests of fresh urine samples included immediate visual dipstick evaluation for protein, blood, leukocytes, nitrites, $\mathrm{pH}$, and specific gravity, with color and clarity being noted. Stored samples were assessed at the central laboratories for protein, red and white blood cells, leukocyte esterase, color, and clarity by automated urinalysis. In addition, at each laboratory, a spot urine albumin:creatinine ratio (ACR) was determined. The ACR was used to detect microalbuminuria because this test has greater sensitivity for detecting low levels of protein than a protein:creatinine ratio and correlates well with the amount of albumin excreted over 24 hours assessed using a 24-hour urine collection. ${ }^{15}$

\section{Study RTG I I4I 37}

All samples were assessed for protein, glucose, ketones, bilirubin, blood, nitrite, urobilinogen, $\mathrm{pH}$, specific gravity, color, clarity, and turbidity using a fully automated system. Microscopic examination was used to determine the presence of leukocytes, erythrocytes, epithelial cells, and casts. Relevant clinical parameters, including creatinine clearance and ACR, were determined using fresh samples. Adverse events, vital

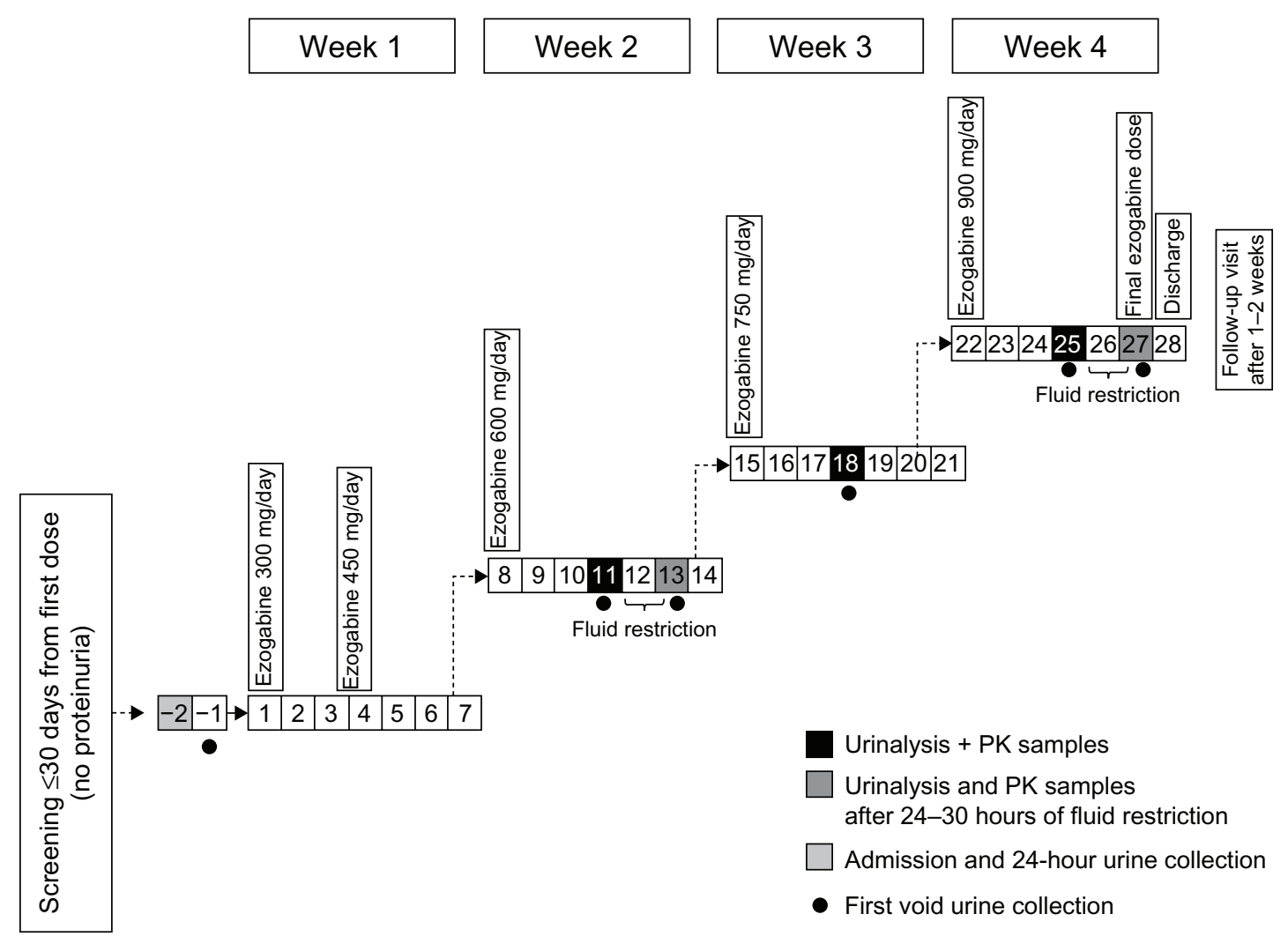

Figure I Design of study RTGI|4|37. Abbreviation: PK, pharmacokinetics. 
signs, and electrocardiographic and clinical chemistry evaluations were determined throughout the study.

\section{Results}

\section{Study 303}

Thirty-five specimens were collected from 16 patients. There was notable variation in clarity, color, and proteinuria between fresh (baseline) and stored samples, and between samples analyzed at different laboratories (Table 1). Proteinuria was detected in $6 \%$ of fresh samples at baseline and $6 \%$ of stored samples analyzed at laboratory B but in $26 \%$ of stored samples analyzed at laboratory A. ACRs were similar for laboratories A and B (mean \pm standard deviation, $15.1 \pm 23.9 \mathrm{mg} / \mathrm{g}$ and $13.5 \pm 22.9 \mathrm{mg} / \mathrm{g}$ creatinine, respectively).

\section{Study RTG I I 4 I 37}

The 33 subjects enrolled (Table 2) all received ezogabine $300 \mathrm{mg} /$ day. As the ezogabine dose was uptitrated, 32 subjects received $450 \mathrm{mg} /$ day and $600 \mathrm{mg} /$ day, 31 received $750 \mathrm{mg} /$ day, and 19 received $900 \mathrm{mg} /$ day. Twenty-four (73\%) subjects completed the study. The study was terminated early because of tolerability concerns with ezogabine $750-900 \mathrm{mg} /$ day, resulting in a maximum treatment duration of 25 days.

\section{Urinalysis}

The results of urinalysis are summarized in Table 3. When fluid intake was not restricted, proteinuria was not detected in fresh samples except for one sample (4\%) on day 25 . For the stored samples, incidences of proteinuria of $0 \%-4 \%$ and $3 \%-8 \%$ were reported on unrestricted fluid intake days in samples containing stabilizers 1 and 2, respectively. After fluid restriction (day 13), proteinuria was detected in two fresh samples (7\%), and in 32\% of stored samples containing stabilizer 1 and in $55 \%-90 \%$ of stored samples containing stabilizer 2. In each case, when protein was detected in the fresh sample, the ACR was within the normal range for the subject's gender (unrestricted fluid, $3.0 \mathrm{mg} / \mathrm{g}$ creatinine; restricted fluid, $14.0 \mathrm{mg} / \mathrm{g}$ and $17.0 \mathrm{mg} / \mathrm{g}$ ).

Table I Incidence of urinalysis abnormalities in Study 303

\begin{tabular}{llll}
\hline & \multicolumn{3}{l}{ Urine samples $(\mathbf{n}=\mathbf{3 5})$} \\
\cline { 2 - 4 } & Baseline & \multicolumn{2}{l}{ Stored laboratory } \\
\cline { 3 - 4 } & & A & B \\
\hline Not clear, $\mathrm{n}(\%)$ & $10(29)$ & $35(100)$ & $16(46)$ \\
Discolored, $\mathrm{n}(\%)$ & $12(34)$ & $10(29)$ & $1(3)$ \\
Protein positive, $\mathrm{n}(\%)$ & $2(6)$ & $9(26)$ & $2(6)$ \\
\hline
\end{tabular}

Table 2 Baseline demographic characteristics for Study RTG I | 4 I 37

\begin{tabular}{ll}
\hline Safety and pharmacodynamic population & Total $(\mathbf{n}=\mathbf{3 3})$ \\
\hline Mean age, years & 47.4 \\
Gender, $\mathrm{n}(\%)$ & \\
$\quad$ Female & $8(24)$ \\
Race, $\mathrm{n}(\%)$ & \\
White/European heritage & $18(55)$ \\
Black/African heritage & $11(33)$ \\
Mean body mass index $\left(\mathrm{kg} / \mathrm{m}^{2}\right)$ & 26.9 \\
\hline
\end{tabular}

On unrestricted fluid intake days, crystals were detected in fresh samples from two subjects. In both cases, the crystals were identified as hyaline casts. Following fluid restriction, crystals were detected in urine from four subjects (calcium oxalate crystals in one subject and hyaline casts in three subjects).

When fluid intake was not restricted, a change in urine color was seen between fresh samples and those stored for 24 and 72 hours, respectively, in $13 \%-23 \%$ and $31 \%-73 \%$ of samples containing stabilizer 1 , and in $0 \%-31 \%$ and $10 \%-22 \%$ of samples containing stabilizer 2 . Following fluid restriction, urine color change was detected in $0 \%$ and $7 \%$ of samples containing stabilizer 1 and in $29 \%$ and $65 \%$ of samples containing stabilizer 2 at 24 and 72 hours, respectively.

No notable changes were observed in other urinalysis parameters in fresh samples when fluid intake was not restricted but, following fluid restriction, red and white blood cells, dipstick occult blood, and haziness were present in a slightly higher proportion of subjects, and average pH was increased (mean 6.1 versus 5.8 at baseline). Fluid restriction was associated with an increase in urine density (Figure 2).

\section{Clinical and laboratory parameters}

With the exception of one subject with an elevated alanine aminotransferase level that was reported as an adverse event, no subject had abnormal hematology or clinical chemistry values, or changes in vital signs or electrocardiograms, that were considered clinically significant.

\section{Safety and tolerability}

The most common adverse events were dizziness and euphoric mood, both reported in $42 \%$ (14/33) of subjects, followed by somnolence $(36 \%, 12 / 33)$ and headache $(33 \%$, $11 / 33)$, as seen in Table 4 . The only urinary tract-related adverse events reported were chromaturia $(18 \%, 6 / 33)$, pollakiuria $(6 \%, 2 / 33)$, and dysuria $(3 \%, 1 / 33)$, all of which resolved spontaneously. 
Table 3 Incidence of urinalysis abnormalities in Study RTG I I I 37

\begin{tabular}{|c|c|c|c|c|c|}
\hline & \multicolumn{5}{|c|}{ Subjects (\%) } \\
\hline & \multirow[t]{2}{*}{ Fresh } & \multicolumn{2}{|c|}{ Stabilizer I } & \multicolumn{2}{|c|}{ Stabilizer 2} \\
\hline & & 24 hours & 72 hours & 24 hours & 72 hours \\
\hline \multicolumn{6}{|l|}{ Protein in urine } \\
\hline \multicolumn{6}{|l|}{ Non-restricted fluid days } \\
\hline Day II (ezogabine 600 mg) & 0 & 3 & 3 & 3 & 3 \\
\hline Day 18 (ezogabine 750 mg) & 0 & 0 & 3 & 3 & 3 \\
\hline Day 25 (ezogabine 900 mg) & 4 & 4 & 4 & 8 & 4 \\
\hline \multicolumn{6}{|l|}{ Restricted fluid day } \\
\hline Day I3 (ezogabine 600 mg) & 7 & 32 & 32 & 90 & 55 \\
\hline \multicolumn{6}{|l|}{ Crystals in urine } \\
\hline \multicolumn{6}{|l|}{ Non-restricted fluid days } \\
\hline Day II (ezogabine 600 mg) & 0 & 3 & 0 & 3 & 3 \\
\hline Day 18 (ezogabine 750 mg) & 3 & 0 & 0 & 0 & 0 \\
\hline Day 25 (ezogabine 900 mg) & 4 & 0 & 4 & 0 & 4 \\
\hline \multicolumn{6}{|l|}{ Restricted fluid day } \\
\hline Day 13 (ezogabine 600 mg) & 13 & 23 & 39 & 0 & 3 \\
\hline \multicolumn{6}{|l|}{ Urine color change } \\
\hline \multicolumn{6}{|l|}{ Non-restricted fluid days } \\
\hline Day II (ezogabine 600 mg) & - & 13 & 31 & 31 & 22 \\
\hline Day 18 (ezogabine 750 mg) & - & 23 & 73 & 17 & 10 \\
\hline Day 25 (ezogabine 900 mg) & - & 20 & 56 & 0 & 16 \\
\hline \multicolumn{6}{|l|}{ Restricted fluid day } \\
\hline Day I3 (ezogabine 600 mg) & - & 0 & 7 & 29 & 65 \\
\hline
\end{tabular}

Ezogabine 300-450 mg/day was reasonably well tolerated, with all reported adverse events being of mild or moderate intensity. An increased incidence of adverse events was reported for ezogabine $600-900 \mathrm{mg} /$ day, which included six of severe intensity. One serious adverse event was reported in the $900 \mathrm{mg}$ /day group: this was a psychotic disorder that resolved spontaneously within an hour and was considered by the principal investigator to be possibly related to the study medication. The subject was withdrawn from treatment and the study was terminated early following this event. Eight other subjects had adverse events leading to withdrawal, including one subject with alanine aminotransferase elevation

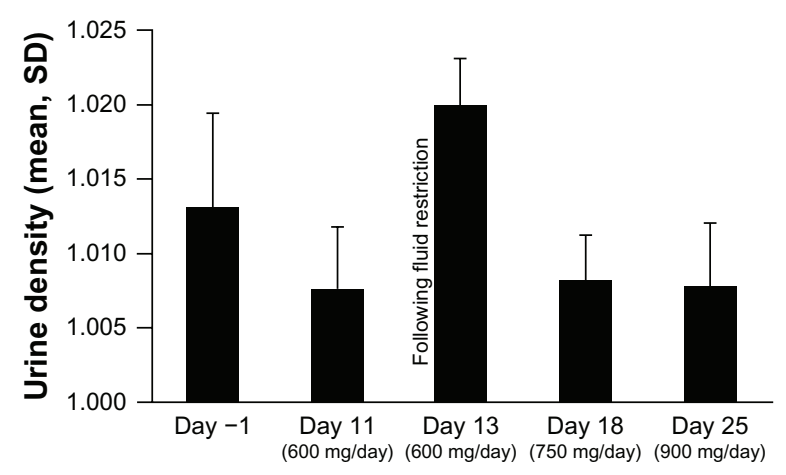

Figure 2 Effect of fluid restriction on the density of urine from healthy volunteers receiving ezogabine in Study RTGI I4I37. Abbreviation: SD, standard deviation. (more than $3 \times$ upper limit of normal), which had resolved at follow-up. There were no deaths during the study.

\section{Discussion}

In a Phase II study in post-herpetic neuralgia, urinalysis results reported from stored, stabilized urine samples indicated that nearly all urine specimens from ezogabine-treated patients were hazy or cloudy, half were abnormally colored, and around one-third tested positive for the presence of protein. In contrast, the majority of patients who received placebo had normal urine color; turbidity increased only marginally, and protein readings remained similar to those at baseline. However, no clinically meaningful changes in renal function were detected, and no adverse events were associated with the urinalysis findings, which resolved completely upon withdrawal of ezogabine. The hypothesis that these urinalysis findings could be attributed to ex vivo processes and urine sample handling procedures was tested in the two studies reported here in which urinalysis was conducted on both freshly voided urine specimens and stored stabilized samples.

Urinalysis performed on fresh specimens indicated that treatment with ezogabine $600-900 \mathrm{mg}$ /day was not associated with clinically significant abnormalities. In Study 303, differences in urinalysis data were noted between fresh 
Table 4 Adverse events occurring in $\geq 2$ subjects by ezogabine dose for Study RTG I |4 I 37

\begin{tabular}{|c|c|c|c|c|c|c|}
\hline \multirow[t]{3}{*}{ Preferred term } & \multicolumn{6}{|c|}{ Ezogabine daily dose } \\
\hline & \multicolumn{6}{|l|}{ n (\%) } \\
\hline & $\begin{array}{l}300 \mathrm{mg} \\
\mathrm{n}=33\end{array}$ & $\begin{array}{l}450 \mathrm{mg} \\
\mathrm{n}=32\end{array}$ & $\begin{array}{l}600 \mathrm{mg} \\
\mathrm{n}=32\end{array}$ & $\begin{array}{l}750 \mathrm{mg} \\
\mathrm{n}=31\end{array}$ & $\begin{array}{l}900 \mathrm{mg} \\
\mathrm{n}=19\end{array}$ & $\begin{array}{l}\text { Total } \\
\mathbf{n}=33\end{array}$ \\
\hline Subject with any drug-related adverse event & $12(36)$ & 14 (44) & $24(75)$ & $18(58)$ & $12(63)$ & $31(94)$ \\
\hline Dizziness & $2(6)$ & $2(6)$ & $7(22)$ & $7(23)$ & I (5) & $14(42)$ \\
\hline Euphoric mood & $6(18)$ & $3(9)$ & $4(\mid 3)$ & I (3) & $2(11)$ & $14(42)$ \\
\hline Somnolence & $3(9)$ & $5(16)$ & I (3) & $3(10)$ & I (5) & $12(36)$ \\
\hline Headache & I (3) & 0 & $4(13)$ & $7(23)$ & $2(I I)$ & II (33) \\
\hline Memory impairment & 0 & I (3) & $5(16)$ & $3(10)$ & 0 & $9(27)$ \\
\hline Oral paresthesia & 0 & I (3) & $2(6)$ & $5(16)$ & 0 & $8(24)$ \\
\hline Confusional state & 0 & 0 & $2(6)$ & $3(10)$ & $3(16)$ & $7(2 I)$ \\
\hline Dysarthria & $2(6)$ & 0 & I (3) & $4(13)$ & 0 & $7(2 I)$ \\
\hline Chromaturia & 0 & 0 & $2(6)$ & $3(10)$ & I (5) & $6(18)$ \\
\hline Disturbance in attention & I (3) & 0 & $2(6)$ & $3(10)$ & 0 & $6(18)$ \\
\hline Dry mouth & 0 & 0 & $2(6)$ & I (3) & $2(11)$ & $5(15)$ \\
\hline Heterophoria & 0 & 0 & $2(6)$ & I (3) & $2(11)$ & $5(15)$ \\
\hline Constipation & I (3) & $2(6)$ & 0 & I (3) & 0 & $4(12)$ \\
\hline Abnormal coordination & 0 & 0 & $2(6)$ & $2(6)$ & 0 & $4(12)$ \\
\hline Nausea & 0 & 0 & $3(9)$ & $2(6)$ & 0 & $4(12)$ \\
\hline Paresthesia & 0 & 0 & I (3) & $3(10)$ & 0 & $4(12)$ \\
\hline Abdominal pain & 0 & I (3) & 0 & $2(6)$ & 0 & $3(9)$ \\
\hline Fatigue & 0 & 0 & I (3) & $2(6)$ & 0 & $3(9)$ \\
\hline Blurred vision & 0 & 0 & $2(6)$ & I (3) & I (5) & $3(9)$ \\
\hline Affect lability & 0 & $2(6)$ & 0 & 0 & 0 & $2(6)$ \\
\hline Arthralgia & 0 & 0 & I (3) & $2(6)$ & 0 & $2(6)$ \\
\hline Ataxia & 0 & 0 & 0 & $2(6)$ & 0 & $2(6)$ \\
\hline Decreased appetite & 0 & 0 & 0 & $2(6)$ & 0 & $2(6)$ \\
\hline Pollakiuria & $2(6)$ & 0 & 0 & 0 & 0 & $2(6)$ \\
\hline
\end{tabular}

and stored/transported samples and between two central laboratories. In Study RTG114137, differences in urinalysis data were observed between fresh and stored samples and with the use of different stabilizers. Most examples of proteinuria, crystal formation, turbidity, and discoloration were detected in specimens stored for 24-72 hours and are considered to be the result of ex vivo processes. The results following fluid restriction clearly indicate that, in the presence of high urine density, addition of a urine stabilizer and delaying urinalysis by 24 hours or more was associated with an increase in positive protein readings. Results from one of the central laboratories used in Study 303 and from stored samples following fluid intake restriction in Study RTG114137 were similar to the findings of the post-herpetic neuralgia study mentioned previously.

The results reported here help to explain the incidence of proteinuria recorded in the post-herpetic neuralgia study. However, there are differences between the studies that should be borne in mind, and the number of patients involved and samples analyzed in the studies reported were limited. Participants in the post-herpetic neuralgia study were older (mean age 62.5 years) than participants in Study RTG114137 (mean age 47.4 years) or the open-label extensions of the Phase III epilepsy studies, including Study 303 (mean age 36.6 years). ${ }^{14}$ In addition, in common with Study 303, but in contrast to Study RTG114137, subjects in the post-herpetic neuralgia study were treated as outpatients. Inpatient treatment has the advantages of allowing close monitoring and control of physical activity and fluid intake, and of diet standardization. The effect of inpatient status on hydration levels can be seen in Study RTG114137, with higher and more variable urine density at study entry than on days 11 , 18, and 25 (Figure 2).

These studies highlight the difficulties in comparing diagnostic test data between clinical studies. Although automated urinalysis has improved accuracy compared with dipsticks, varying sensitivities, specificities, and high rates of false-positive results can occur. ${ }^{16-18}$ The light-refractory properties of the bilirubin-like crystals observed in the urine of patients receiving ezogabine, together with color formation in aqueous solutions of ezogabine, may contribute to the turbidity and discoloration reported in stored samples. In the studies described here and in the post-herpetic neuralgia study, the lack of clinically relevant changes in 
laboratory parameters, such as serum creatinine and blood urea nitrogen, supports the lack of a real renal effect of the study medication.

The adverse event profile for ezogabine reported here is consistent with that reported previously, including the association of ezogabine $600-900 \mathrm{mg} /$ day with a high frequency of central nervous system-related adverse events..$^{5,6,14}$ To limit the duration of exposure of healthy volunteers to ezogabine, the schedule for uptitration to $600 \mathrm{mg}$ /day was more rapid in Study RTG114137 than that used in the pivotal controlled trials and recommended for clinical use. ${ }^{4}$

In conclusion, a detailed investigation of the impact of the interval between urine collection and analysis, patient hydration levels, and the type of preservative used for urine specimen storage at a central laboratory has demonstrated the importance of performing urinalysis on fresh urine samples from patients receiving ezogabine if the incidence of falsepositive proteinuria findings is to be minimized.

\section{Disclosure}

The studies were conducted by Valeant Pharmaceuticals International and GlaxoSmithKline. Editorial support in the form of writing and collating author comments was provided by Dana Fox (Caudex Medical Inc, New York, NY) and funded by GlaxoSmithKline and Valeant Pharmaceuticals International. $\mathrm{NB}, \mathrm{SD}, \mathrm{MB}, \mathrm{CE}$, and $\mathrm{SJ}$ have received personal compensation from GlaxoSmithKline as employees, and own stock and stock options from GlaxoSmithKline.

\section{References}

1. Rundfeldt $\mathrm{C}$, Netzer R. The novel anticonvulsant retigabine activates M-currents in Chinese hamster ovary-cells transfected with human KCNQ2/3 subunits. Neurosci Lett. 2000;282:73-76.

2. Ferron GM, Paul J, Fruncillo R, et al. Multiple-dose, linear, doseproportional pharmacokinetics of retigabine in healthy volunteers. J Clin Pharmacol. 2002;42:175-182.

3. Borlak J, Gasparic A, Locher M, Schupke H, Hermann R. $\mathrm{N}$-Glucuronidation of the antiepileptic drug retigabine: results from studies with human volunteers, heterologously expressed human UGTs, human liver, kidney, and liver microsomal membranes of Crigler-Najjar type II. Metabolism. 2006;55:711-721.
4. GlaxoSmithKline. Potiga ${ }^{\mathrm{TM}}$ Prescribing Information. Available from: http://us.gsk.com/products/assets/us_potiga.pdf. Accessed December 6, 2012.

5. Porter RJ, Partiot A, Sachdeo R, Nohria V, Alves WM. Randomized, multicenter, dose-ranging trial of retigabine for partial-onset seizures. Neurology. 2007;68:1197-1204.

6. Brodie MJ, Lerche H, Gil-Nagel A, et al. Efficacy and safety of adjunctive ezogabine (retigabine) in refractory partial epilepsy. Neurology. 2010;75:1817-1824.

7. French JA, Abou-Khalil BW, Leroy RF, et al. Randomized double-blind placebo-controlled trial of ezogabine (retigabine) in partial epilepsy. Neurology. 2011;76:1555-1563.

8. Streng T, Cristoph T, Andersson KE. Urodynamic effects of the K+ channel (KCNQ) opener retigabine in freely moving conscious rats. J Urol. 2004;172:2054-2058.

9. Greenwood IA, Ohya S. New tricks for old dogs. KCNQ expression and role in smooth muscle. Br J Pharmacol. 2009;156:1196-1203.

10. Rode F, Svalo J, Sheykhzade M, Ronn LC. Functional effects of the KCNQ modulators retigabine and XE991 in the rat urinary bladder. Eur J Pharmacol. 2010;638:121-127.

11. Brickel N, Gandhi P, VanLandingham K, Hamond J, DeRossett S. The urinary safety profile and secondary renal effects of retigabine (ezogabine): a first-in-class antiepileptic drug that targets KCNQ (K(v)7) potassium channels. Epilepsia. 2012;53:606-612.

12. Fogazzi GB. Crystalluria: a neglected aspect of urinary sediment analysis. Nephrol Dial Transplant. 1996;11:379-387.

13. Simerville JA, Maxted WC, Pahira JJ. Urinalysis: a comprehensive review. Am Fam Physician. 2005;71:1153-1162.

14. Gil-Nagel A, Brodie MJ, Leroy R, et al. Safety and efficacy of ezogabine (retigabine) in adults with refractory partial-onset seizures: interim results from two ongoing open-label studies. Epilepsy Res. 2012;102: $117-121$.

15. NICE 2008. Chronic kidney disease. Early identification and management of chronic kidney disease in adults in primary and secondary care. Available from: http://www.nice.org.uk/nicemedia/ live/12069/42117/42117.pdf. Accessed January 2, 2013.

16. Saudan PJ, Brown MA, Farrell T, Shaw L. Improved methods of assessing proteinuria in hypertensive pregnancy. Br J Obstet Gynaecol. 1997;104:1159-1164.

17. Kyle PM, Fielder JN, Pullar B, Horwood LJ, Moore MP. Comparison of methods to identify significant proteinuria in pregnancy in the outpatient setting. BJOG. 2008;115:523-527.

18. National Collaborating Centre for Chronic Conditions. Chronic Kidney Disease: National Clinical Guideline for Early Identification and Management of Adults in Primary and Secondary Care. London, UK: Royal College of Physicians; 2008.
Therapeutics and Clinical Risk Management

\section{Publish your work in this journal}

Therapeutics and Clinical Risk Management is an international, peerreviewed journal of clinical therapeutics and risk management, focusing on concise rapid reporting of clinical studies in all therapeutic areas, outcomes, safety, and programs for the effective, safe, and sustained use of medicines. This journal is indexed on PubMed Central, CAS,

\section{Dovepress}

EMBase, Scopus and the Elsevier Bibliographic databases. The manuscript management system is completely online and includes a very quick and fair peer-review system, which is all easy to use. Visit http://www.dovepress.com/testimonials.php to read real quotes from published authors. 